\title{
Test of Universality in Anisotropic 3D Ising Model
}

\author{
M. A. Sumour, Physics Department, Al-Aqsa University, P.O.4051, Gaza, \\ Gaza Strip, Palestinian Authority, msumoor@yahoo.com \\ D. Stauffer,Institute for Theoretical Physics, Cologne University, D-50923 \\ Köln, Germany, stauffer@thp.uni-koeln.de \\ M.M. Shabat, Physics Department, Islamic University, P.O.108, Gaza, \\ Gaza Strip, Palestinian Authority, shabat@mail.iugaza.edu \\ A.H. El-Astal, Physics Department, Al-Aqsa University, P.O.4051, Gaza, \\ Gaza Strip, Palestinian Authority, a_elastal@yahoo.com
}

Abstract:

Chen and Dohm predicted theoretically in 2004 that the widely believed universality principle is violated in the Ising model on the simple cubic lattice with more than only six nearest neighbours. Schulte and Drope by Monte Carlo simulations found such violation, but not in the predicted direction. Selke and Shchur tested the square lattice. Here we check only this universality for the susceptibility ratio near the critical point. For this purpose we study first the standard Ising model on a simple cubic lattice with six nearest neighbours, then with six nearest and twelve next-nearest neighbours, and compare the results with the Chen-Dohm lattice of six nearest neighbours and only half of the twelve next-nearest neighbours. We do not confirm the violation of universality found by Schulte and Drope in the susceptibility ratio.

\section{Introduction}

To study the critical phenomena of any system, all systems are divided [1] into a small number of universality classes. They are characterized by the dimensionality of the space and the number of components of the order parameter. Within a certain universality class, the universal quantities (critical exponents, amplitude ratios, and scaling functions) are independent of microscopic details, such as the particular type of interactions or lattice structure.

Once the universal quantities of a universality class are known, the asymptotic critical behavior of very different systems (e.g. fluids and magnets) is believed to be known completely provided that only two nonuniversal amplitudes and the non-universal critical temperature $T_{c}$ are given. 


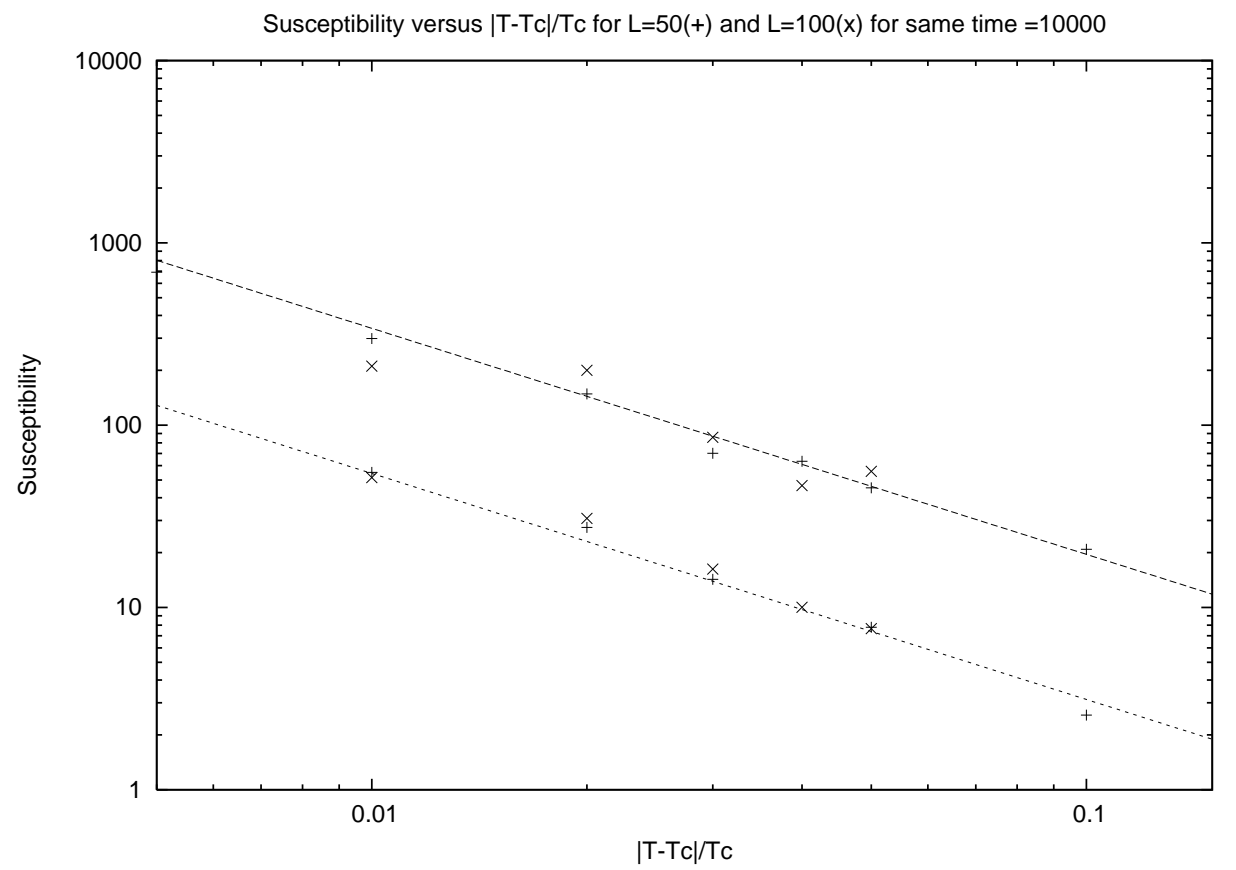

Figure 1: Susceptibility versus temperature difference for $L=50$ and 100 for 6 nearest neighbours as log-log plot. The upper data correspond to $T>T_{c}$, the lower to $T<T_{c}$. Always 1000 iterations were made. The straight lines in Figs.1,3,4 have the theoretical slope -1.24 .

Here the 3D Ising universality class is considered by studying the susceptibility of nearest neighbour Ising model (NN model), and the next-nearest neighbour Ising model (NNN model) with only six NNN. Here some deviations from universality were predicted [2] and partially confirmed [3,4]. Also with directed interactions problems occur in the Ising model $[5,6,7]$. Note that Chen and Dohm [2] made no prediction on the susceptibility ratio [8] and thus are not directly tested in the present paper, which only checks on a result of [3].

In the following section, we study the susceptibility for the NN model and the ratio of the susceptibilities above to below $T_{c}$, and then we simulate the NNN model for 3D Ising model for different interaction ratios $R$, all with Glauber kinetics. Periodic and helical boundary conditions were used throughout. The susceptibilities were calculated through the fluctuations of 


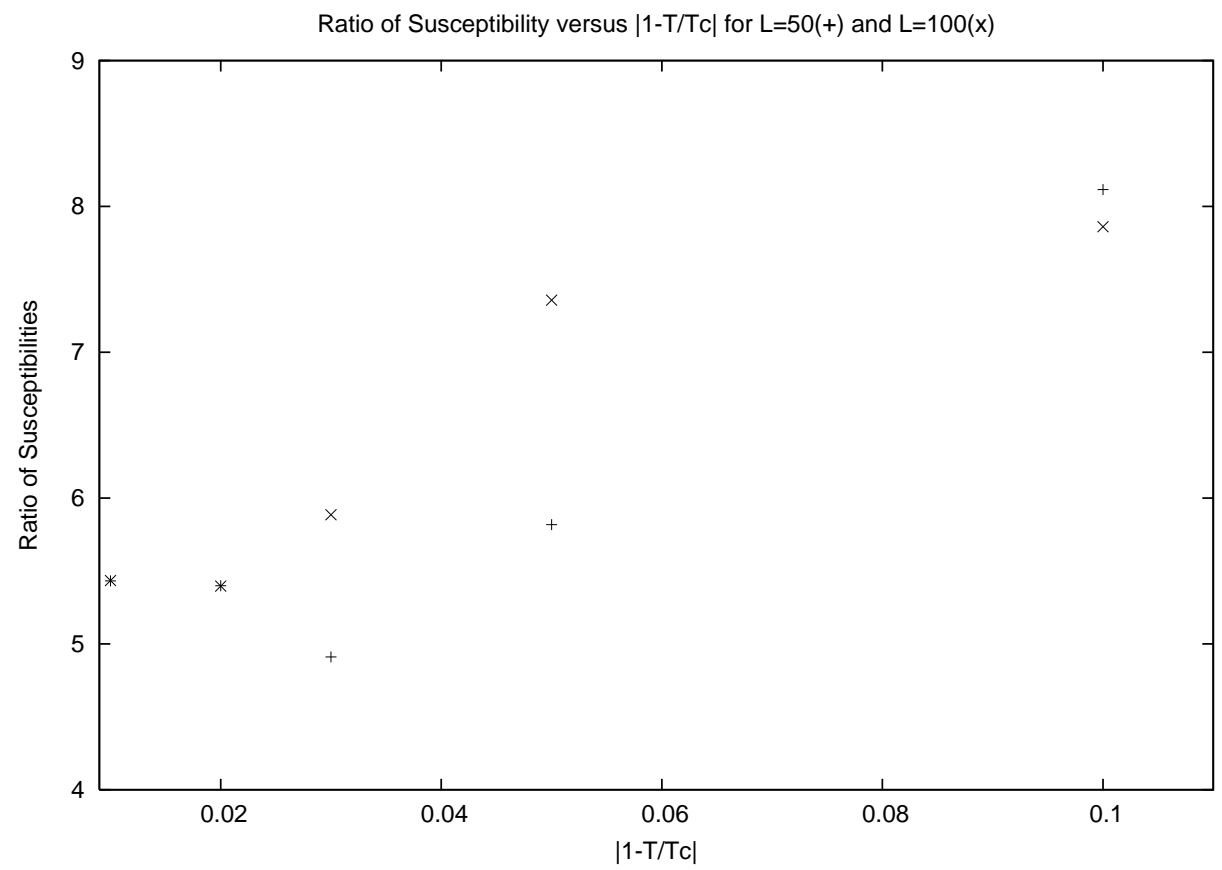

Figure 2: Ratio of susceptibilities above to below $T_{c}$, versus $\left|1-T / T_{c}\right|$, for the two lattices $L=50$ and 100 for 6 nearest neighbours.

the magnetisation while Ref.3 used the those of the absolute value of the magnetisation.

\section{NN Ising Model for 3D}

To test the variation of susceptibility within the 3D Ising universality class, we choose first the NN model without external field on the simple cubic lattice of $L \times L \times L$ spins. Each lattice site has 6 nearest neighbours. We take two sizes of lattices ( $L=50$ and 100), where $L$ is the size of lattice, and then plot the susceptibility versus $\left|T-T_{c}\right| / T_{c}$ double-logarithmically in Fig.1. As we see there is little difference in the susceptibility between the two lattice sizes.

Fig. 2 shows the ratio of susceptibilities above and below $T_{c}$ for both lattices, $L=50$ and 100, at the same distance from $T_{c}$, versus this distance. It is consistent with the well established value near 5, and thus confirms our simulation and analysis methods. 

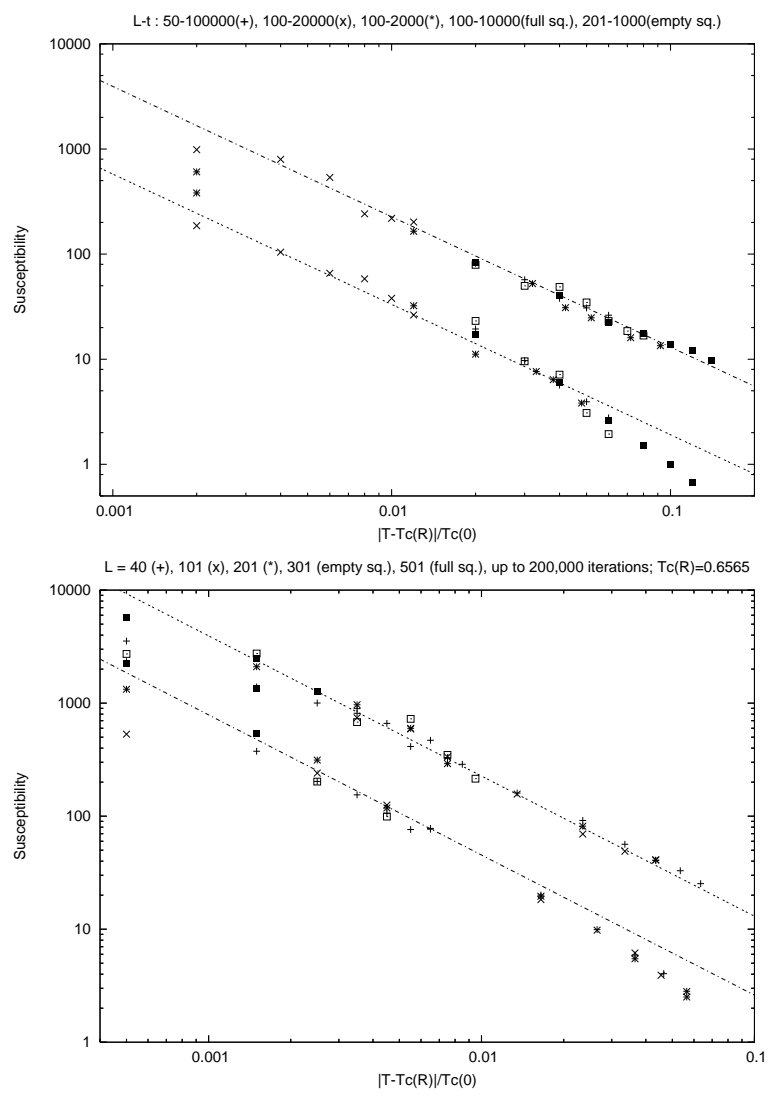

Figure 3: As Fig. 1 but with 6 NNN neighbours added to the 6 NN neighbours; interaction ratio $R=-0.237$. The lower part includes larger systems.

\section{NNN Ising Model for 3D}

Also in the case of $6 \mathrm{NN}$ and $12 \mathrm{NNN}$ interactions of equal strength, we find a susceptibility ratio near 5 (not shown). Furthermore we study the universality of susceptibilities in the zero field NNN Ising model with antiferromagnetic anisotropic next-nearest neighbour coupling, added to the ferromagnetic NN coupling with a negative ratio $R$ of NNN to NN interaction strength. The anisotropic NNN Ising model [2] is established by considering only 6 of the 12 next-nearest neighbours being effective for NNN interaction, and the other 6 NNN having no interaction. The interacting NNN have the position differences $\pm(1,1,0), \pm(1,0,1), \pm(0,1,1)$ on a simple cubic lattice. And we do simulations for different sizes of lattices, times and temperatures, 


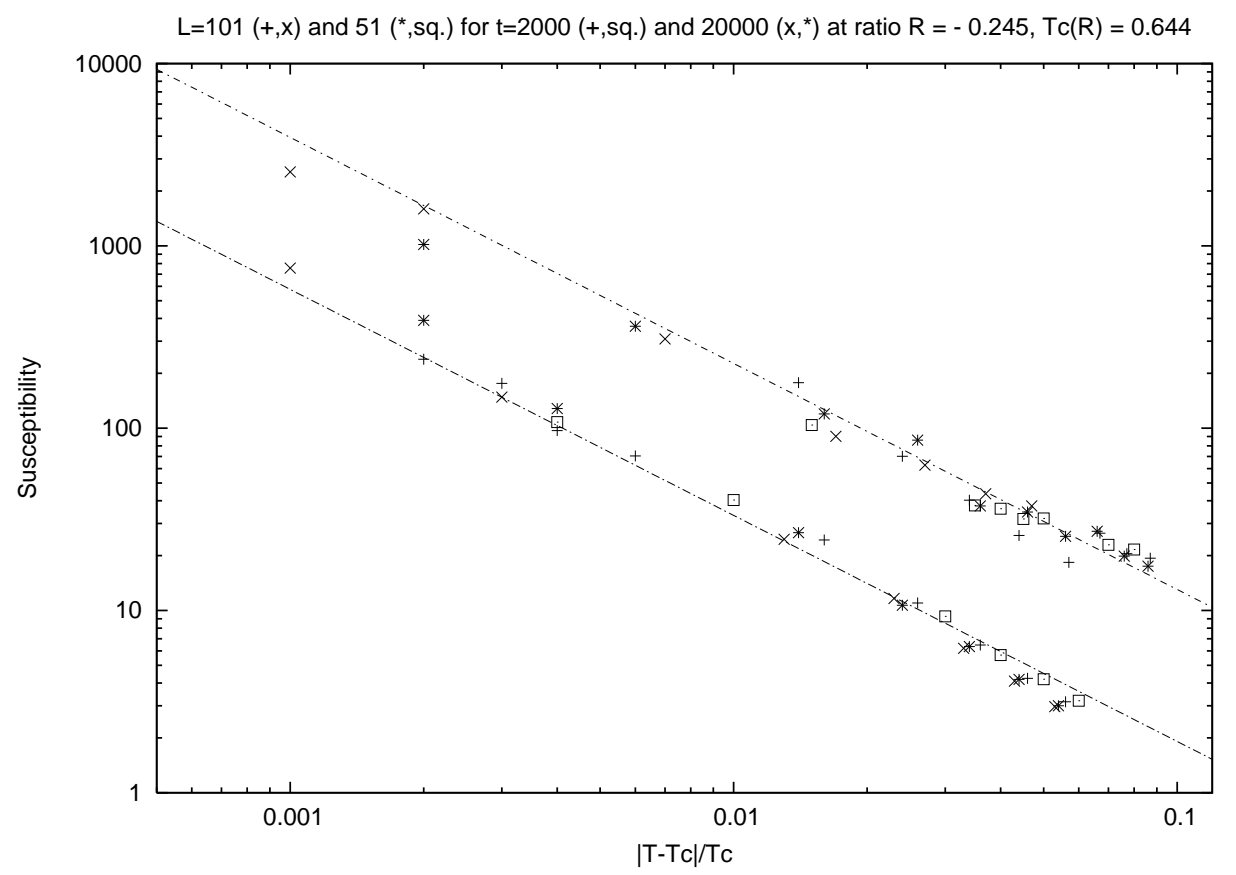

Figure 4: As Fig.3 but for $R=-0.245$, as in [3].

and same coupling ratios $R=-0.237$ and -0.245 as used as the extreme cases in [3]. Sizes between 40 and 501 and times between 1000 and 200,000 are given in the headlines of Fig.3. The critical temperatures were determined by the maxima of the susceptibility, like $0.656_{5}$ at $\mathrm{R}+-0.237$, in units of $T_{c}$ for the NN model.

Fig. 3 shows the susceptibility versus the temperature difference to $T_{c}(R)$ as a log-log plot. Since the resulting susceptibility ratio is consistent with the one shown for only nearest neighbours and not consistent with the increased value found by Schulte and Drope [3], we repeated the simulations at a different place using a different computer and larger lattices up to $L=501$; the results in Figs. 3 and 4 confirm the universality of the susceptibility ratio.

In general one lattice only was simulated, and the errors thus can best be seen by comparing our different symbols at the same temperature, corresponding to different times and different size; then one also sees the systematic errors. Fig. 3b shows that the data closest to $T_{c}$ are too much influenced by these systematic errors, while the two upper full squares in that Fig.3b 
at a relative temperature difference of 0.0015 correspond to $L=501$ with 100,000 (bad) and 200,000 (better) iterations. The critical temperature was determined from the maximum of the susceptibility.

Ref.3 only simulated temperatures $3 \%$ above and below $T_{c}$ which is in the asymptotic regime for the nearest-neighbour case Fig. 1 but not for the more complicated lattice below $T_{c}$ in Fig.3. Perhaps that is the reason why they got too high susceptibility ratios; our data using a wide range of temperature differences show that $1 \%$ difference would have been better and that at this smaller difference no reliable deviation from a universal ratio exists.

\section{Conclusion}

The susceptibility ratio, as we see from our simulations for different $R=$ $0,-0.237,-0.245$, keeps the universality of the 3D Ising model above and below the critical temperature. For 6 nearest neighbours, for $6+12$ nearest next-nearest neighbours and for $6+6$ nearest neighbours, there is no reliable difference for the susceptibility ratio, contradicting [3] but compatible with the standard ratio of $4.7_{5}[9]$. We did not attempt to simulate the more subtle universality questions connected with Binder cumulants $[2,4]$.

We thank W. Selke for comments on the manuscript. DS thanks Universidade Federal Fluminense in Brazil for its hospitality during the time of his simulations.

\section{References}

[1] K. G. Wilson, Phys. Repts. 12, 75 (1974); Rev. Mod. Phys. 47, 773 (1975).

[2] X. S. Chen and V. Dohm, Phys. Rev. E 70, 056136 (2004).

[3] M. Schulte and C. Drope, Int. J. Mod. Phys. C 16, 1217 (2005).

[4] W. Selke and L.N. Shchur, J. Phys. A 39, L 739 (2005).

[5] M. A. Sumour and M. M. Shabat, Int. J. Mod. Phys. C 16, 585 (2005);

[6] M. A. Sumour, M. M. Shabat and D. Stauffer, Islamic University Conference, March 2005; to be published in the university magazine.

[7] F. W. S. Lima and D. Stauffer, Physica A 359, 423 (2005).

[8] D.D. Betts, A.J. Guttmann, G.S. Joyce, J. Phys. C 4, 1994 (1971).

[9] M. Hasenbusch, Int. J. Mod. Phys. V 12, 911 (2001), eq.(158) and table 16. 